\title{
Recent Advances and Future Opportunities to Address Challenges in Offloading Diabetic Feet: A Mini-Review
}

\author{
Ryan T. Crews $^{a}$ Abagayle L. King ${ }^{b}$ Sai V. Yalla ${ }^{a}$ Noah J. Rosenblatt ${ }^{a}$ \\ ${ }^{a}$ Center for Lower Extremity Ambulatory Research (CLEAR), Dr. William M. Scholl College of Podiatric Medicine, Rosalind \\ Franklin University of Medicine and Science, North Chicago, IL, USA; ${ }^{b}$ Lake Forest College, Lake Forest, IL, USA
}

\author{
Keywords \\ Diabetes · Diabetic foot ulcer · Offload · Footwear · Physical \\ activity
}

\begin{abstract}
Diabetic foot ulcers (DFU) are a substantial dilemma for geriatric individuals with diabetes. The breakdown in tissue associated with DFU is typically a result of repetitive cycles of physical stress placed on the feet during weight-bearing activity. Accordingly, a key tenet in healing as well as preventing DFU is the use of offloading footwear to redistribute physical stress away from high stress locations such as bony prominences. Over the last several years there has been a substantial amount of effort directed at better understanding and implementing the practice of offloading. A review of this work as well as relevant technological advances is presented in this paper. Specifically, we will discuss the following topics in association with offloading diabetic feet: achieving optimal offloading, dosing activity/physical stress, thermal monitoring to detect preulcerative tissue damage, adherence with offloading devices, and optimizing the user experience. In addition to presenting progress to date, potential directions for further advancement are discussed.
\end{abstract}

(c) 2018 S. Karger AG, Basel

\section{KARGER}

(c) 2018 S. Karger AG, Basel

E-Mail karger@karger.com

www.karger.com/ger

\section{Introduction}

Diabetic foot ulcers (DFU) represent a significant global challenge for older individuals [1]. Approximately $18-34 \%$ of individuals with diabetes will develop a DFU within their lifetime [2]. Infection and lower limb amputation are serious secondary concerns as well as the fact that life expectancy declines following the development of a DFU [2]. The disproportionately high impact of this condition on the elderly was highlighted by a recent health care services and outcomes study that reported a mean age of $67 \pm 16$ years for its 2.5 million DFU cases and $67 \pm 16$ years for its 4.2 million diabetic foot infection cases [3]. The economic burden of DFU upon older adults is enormous. In the USA, Medicare fees for service beneficiaries (typically aged 65 years or older) with DFU are approximately 3 times higher for care than those for diabetic patients without a prevalent DFU [4]. Although acute forms of trauma can result in DFU, typically DFU form in response to repetitive cycles of physical stress induced by weight-bearing physical activity which are imperceptible due to the presence of diabetic peripheral neuropathy [5]. Therefore, a central tenet to healing active DFU and avoiding future ulcers is "offloading" areas

Ryan T. Crews

CLEAR, Rosalind Franklin University of Medicine and Science

3333 Green Bay Road

North Chicago, IL 60077 (USA)

E-Mail ryan.crews@ rosalindfranklin.edu 


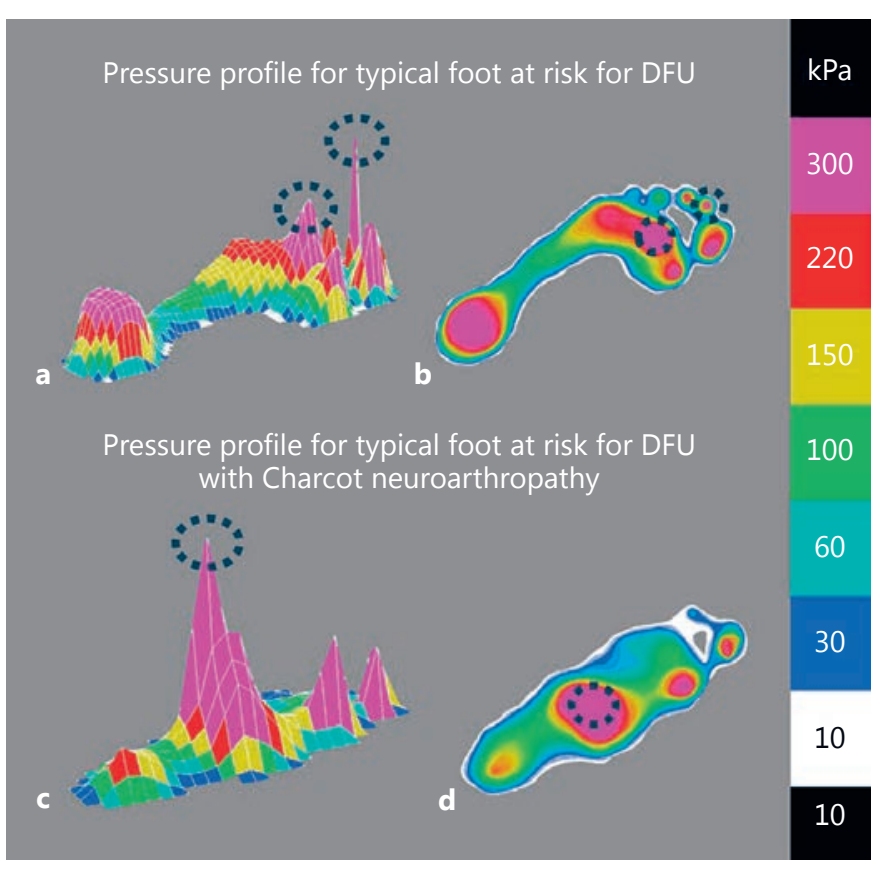

Fig. 1. Plantar pressure profiles of diabetic feet at risk of diabetic foot ulcers (DFU). Dotted circles show regions of high pressure at risk for DFU. a 3-D pressure profile of typical at-risk foot. b 2-D pressure profile of typical at-risk foot. c 3-D pressure profile of atrisk foot with Charcot neuropathy deformity. $\mathbf{d} 2$-D pressure profile of at-risk foot with Charcot neuropathy deformity.

of high stress on the plantar surface of the feet (Fig. 1) via specialized footwear $[6,7]$.

Numerous changes to the lower extremities of individuals with diabetes are associated with disease progression and aging $[8,9]$. These changes often enhance the need for appropriate offloading. For example, a review by Bus [9] provides evidence that common deformities of the diabetic foot, including claw/hammer toes, excessive callus formation, and limited joint mobility of the diabetic foot, are associated with increased plantar pressure. The less common but "most devastating" structural abnormality of the diabetic foot, Charcot neuroarthropathy, also greatly increases plantar pressure at the site of deformities (Fig. 1) [9]. In addition to deformities, muscle atrophy in combination with glycosylation of soft tissues of the foot (e.g., tendons and fat pads), when coupled with peripheral neuropathy in patients with diabetes, can alter biomechanics of gait, including generation of breaking and propulsive forces, and in turn impact plantar pressures [8].

In recent years there has been much effort devoted to improving offloading of diabetic feet. This review will

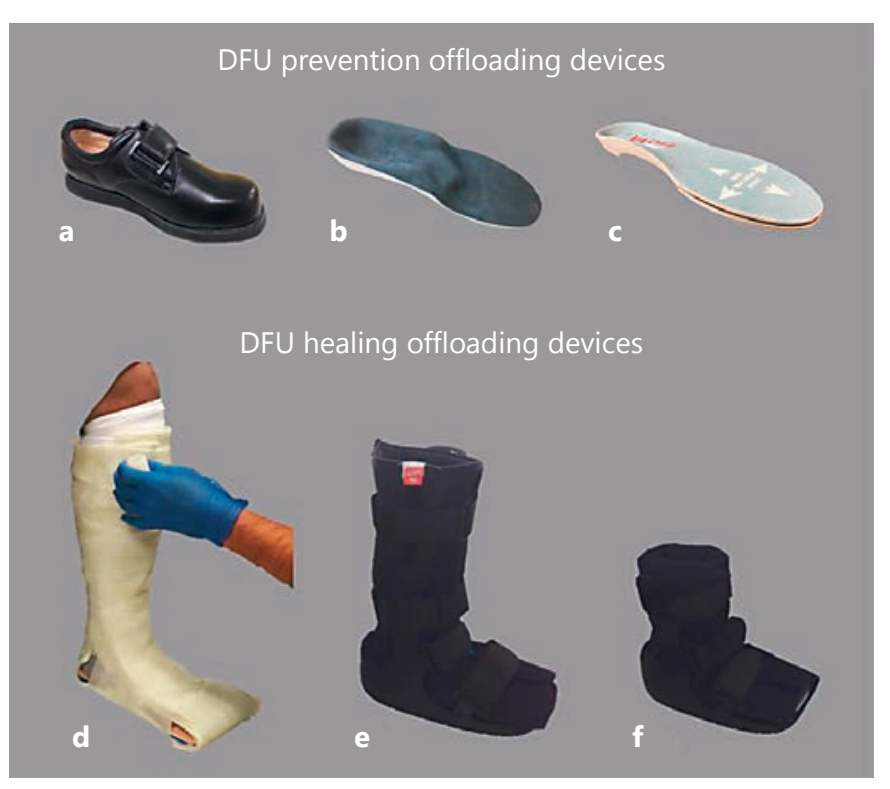

Fig. 2. Representative offloading devices. a Extra depth shoe. b Custom-made orthotic. c Prefabricated orthotic. d Total contact cast. e Removable cast walker (knee-height). f Removable cast walker (ankle-height). DFU, diabetic foot ulcers.

cover recent advances in offloading as well as potential advances on the near horizon. Specifically, it will cover the following important challenges associated with offloading diabetic feet: adherence, achieving optimal offloading, dosing activity/physical stress, monitoring tissue health, and optimizing the user experience. Given that devices used to heal active DFU typically are much more substantial in size and impact upon lower extremity functionality than devices for preventing DFU (Fig. 2), when possible, the two types of devices will be discussed independently.

\section{Achieving Optimal Offloading}

Offloading devices for treating DFU such as total contact casts and removable cast walkers are able to provide over a $90 \%$ reduction in forefoot peak pressure relative to barefoot walking [10]. Due to their more diminutive nature and reduced impact upon gait, offloading footwear for preventing DFU provide lower reductions in plantar pressure than devices used to treat DFU. Thus, offloading 
devices for preventing DFU have had a larger margin for improvement in terms of offloading capacity. Over the last decade, the art of constructing custom-made preventative offloading footwear has benefitted from the addition of objective plantar pressure assessments. Two different approaches have been successfully utilized. One method is to incorporate plantar pressure measurements directly into a computerized milling machine's manufacturing of insoles [11]. The other method is to measure the plantar pressure on users' feet while wearing traditionally manufactured footwear and then have a shoe technician modify the footwear based upon the observed pressure values [12]. Multiple cycles of plantar pressure measurements and subsequent footwear modifications are possible with this method. Although both methods of incorporating plantar pressure measurements into the production of offloading footwear failed to demonstrate reductions in preulcerative lesions compared to traditional methods, they both led to decreases in the recurrence of DFU $[11,12]$. However, there is one caveat to the reduction in DFU. As discussed in the adherence section of this paper, the study utilizing plantar pressure assessments to guide footwear modification(s) only demonstrated a reduction in DFU recurrence when focusing on subjects who adherently wore their offloading footwear [12]. The reduction in DFU was not significant when assessing all participants in the study.

In addition to optimizing offloading devices by reducing plantar pressure, some work has aimed at reducing shear stress on the feet. Previous work has suggested that shear-reducing insoles are helpful for preventing DFU in high-risk persons [13]. In contrast, a study on active DFU that compared a removable cast walker containing a shear-reducing foot bed to two other offloading modalities found the shear-reducing cast walker yielded the lowest healing and highest rate of attrition [14]. However, a lack of offloading adherence data from the study presents a challenge to interpreting the results.

\section{Thermal Monitoring for DFU Prevention}

A second focus of recent technological advances has been the identification of preulcerative damage to the plantar tissue of the feet. A variety of thermal assessments are being used to identify preulcerative inflammation in the feet. Regular monitoring of foot temperature has been demonstrated to be predictive of forthcoming DFU [15], and can be used to warn individuals to decrease their physical activity engagement to lessen their likelihood of developing a DFU [16]. The general premise to these interventions is that an inflammatory response is initiated in foot regions that are subjected to excessive physical stress. If a specific site on one foot is warmer than the equivalent location on the contralateral foot, the warm site is likely reflective of preulcerative inflammation. In order to halt ulcer progression, patients are advised to reduce their physical activity if they note a "hot spot." Once temperatures normalize between the two feet, patients are able to resume their normal activities.

The most studied way to assess foot temperatures is through handheld thermometers. Studies evaluating such devices have found that $67-88 \%$ of subjects are highly adherent (with differing definitions of "high") to monitoring their temperatures $[16,17]$. The importance of adherently measuring temperatures was highlighted by a 2007 study [16]. It found that $80 \%(4 / 5)$ of participants who developed a DFU after being assigned to a temperature monitoring group did not comply with measuring their foot temperatures. More recently, a telemedicine system utilizing a floor mat with embedded temperature sensors was evaluated [15]. This simple-to-use system was designed to require no configuration or setup by the users who simply had to step on the mat with both feet for $\sim 20 \mathrm{~s}$. The system then compared the temperature profile of the two feet. Using a threshold of $\geq 2.22^{\circ} \mathrm{C}$ difference between corresponding sites on opposite feet, the mat correctly predicted $97 \%$ of DFU with an average lead time of 37 days. Adherence to the mat was high with $86 \%$ of participants using the mat at least 3 times per week, and average use was 5 times per week. Despite several promising outcomes, the study results also highlighted a drawback to thermal monitoring. In contrast to other studies [16, 17], this study did not utilize temperature data to prevent DFU through behavioral modification and consequently provided a straightforward look at the occurrence of false positives. The threshold of $2.22^{\circ} \mathrm{C}$, which provided $97 \%$ sensitivity, yielded only $43 \%$ specificity. Increasing the threshold value resulted in improvements in specificity paired with declines in sensitivity. Under the standard intervention paradigm for monitoring temperatures of the feet, false positives would result in a user being unnecessarily advised to reduce their physical activity level.

In contrast to the traditional method of taking temperature measurements at either one or several discrete times per day, researchers [18] and private companies are developing smart socks to measure foot temperatures. In addition to being simple to use, temperature-monitoring socks offer the opportunity for continuous temperature measurement throughout the day. This may result in ear- 
lier detection of inflammation and may also identify tissue damage as it is occurring by detecting changes in temperature due to frictional stress placed on the feet during walking. A potential future alternative to instrumented socks is the development of smart shoes/insoles to monitor temperature on the plantar surface of the feet. In addition to identifying damage to the feet, plantar thermal sensors could provide the additional benefit of monitoring footwear adherence (use) as has been done with thermal sensors placed elsewhere in footwear [19].

\section{Dosing Physical Activity and Stress to the Foot}

Another historical challenge associated with preventing DFU has been the identification of specific thresholds of the amplitude (e.g., plantar pressure) and volume (steps taken/time spent standing) of stress the diabetic foot can tolerate. Traditional thought was that at-risk individuals who developed DFU were more active than those who did not develop DFU. However, research has demonstrated that the relationship between activity and DFU is not straightforward. Maluf and Mueller [20] compared physical activity and "cumulative plantar tissue stress" between two groups of patients with diabetic peripheral neuropathy. One group had a history of DFU and the other did not. Cumulative plantar tissue stress was calculated as the product of mean daily strides captured by an activity monitor during a 7-day monitoring period, and the plantar pressure time integral was measured while subjects walked across a 7.6-m walkway. Subjects with a history of DFU accumulated less daily stress than subjects without a history of previous DFU. A subsequent prospective study by Armstrong et al. [21] provided some additional insight into the association between physical activity and DFU formation. The group utilized computerized activity monitors to prospectively monitor the physical activity patterns of 100 patients at risk of DFU. In line with the work by Maluf and Mueller [20], they found that the patients who ulcerated had significantly lower average daily activity levels. However, they also noted that the patients who developed DFU demonstrated significantly greater day-to-day variability in their activity levels. Taken together, these studies suggest low physical activity levels paired with periodic bursts in activity as a mechanism for DFU formation. It is noteworthy that one published study found that greater day-today step count variability was associated with a small but statistically significant protective effect (odds ratio 0.93, 95\% CI: 0.89-0.99) [22]. One potential explanation for this differing finding is that study step count variability was calculated from a single 7-day monitoring period within the 18-month protocol that was not temporally linked to ulcer occurrence. Thus, variability in the days preceding ulceration was not captured in this study.

Collectively, the data to date supports the application of the tissue stress theory [23] to the pathomechanics of DFU formation. Application of the theory suggests that the skin and soft tissue of the feet develop decreased stress tolerance in individuals at risk of DFU who maintain low daily activity levels. When those individuals subsequently have a spike in activity (increased variability) their foot is unable to tolerate the increased stress and a DFU forms. In contrast, individuals who maintain consistently higher levels of physical activity have tissue that is conditioned to tolerate a higher level of stress. Under the theory, individuals who gradually increase their physical activity should benefit from gradually increased stress tolerance. However, individuals who excessively exceed their normal physical stress levels will encounter tissue injury or death.

Armstrong and Boulton [24] postulated nearly 20 years ago that it might be possible to prescribe physical activity "dosages" to at-risk patients. While standardized physical activity recommendations to reduce DFU risk that are based on cumulative tissue stress remain elusive due to individual differences in stress tolerance, there are opportunities to incorporate technologies into offloading footwear to monitor and/or prescribe personalized modifications to physical activity. Efforts are currently underway to commercialize plantar pressure measurement systems for monitoring stress applied to the feet and providing this information to both patients and care providers [18]. At present, these systems are being developed as smart insoles and textiles. However, in the future such technology could be integrated directly into offloading devices to help patients manage daily plantar stresses. In addition to monitoring stresses placed upon the feet throughout the day, advances in physical activity monitors allow individuals to capture information beyond step counts alone. A 2010 study by Najafi et al. [25] found that patients at risk of DFU spent twice as much time per day loading their feet while standing still as opposed to walking. In addition to better capacities to classify and quantify physical activity, efforts are underway to monitor the context in which physical activity is conducted (e.g., indoors/outdoors or home/away from home). A recent investigation validated methodology to integrate GPS data with physical activity data in both patients at risk of DFU and patients with active DFU [26]. Future applications of 
such methodology will likely include the creation of smart phone apps that provide activity recommendations (increase or decrease) based upon a user's current location and past history when in similar environments. Through improved monitoring of foot loading and physical activity patterns, patients and providers will be better able to set personalized activity prescriptions.

Once a DFU actually forms, there is uncertainty regarding the need to advise activity reduction. Conventional wisdom dictates that once an individual exceeds their tissue's tolerance level and develops a DFU, they should limit activity as much as possible to allow the DFU to heal. However, some recent research suggests prescribed inactivity may be unnecessary when offloading footwear appropriate for an active DFU is provided [27]. A secondary analysis of data from a previously published study [28] indicated that weight-bearing activity may not need to be purposefully avoided [27]. In the secondary analysis Vileikyte et al. [27] found that daily step count and offloading adherence level (see Offloading Adherence) were each positively associated with wound healing in subjects with DFU who were provided with offloading footwear intended for healing active DFU (primarily knee-high removable cast walkers). However, offloading adherence was a stronger predictor of DFU healing. The possibility that sufficiently offloaded DFU benefit from physical activity is plausible in light of a growing body of literature suggesting that exercise promotes wound healing in older and obese populations [29]. Thus, recommending inactivity may be unnecessary for appropriately offloaded DFU and may actually be detrimental. However, this concept is not without controversy. Another recent study had some contradictory findings. Najafi et al. [30] randomized 49 patients with active DFU to receive either a removable cast walker or the same cast walker rendered irremovable for the offloading of their wounds. They found negative associations between weight-bearing physical activity and healing. Due to a lack of offloading adherence monitoring in the study, it is not possible to determine whether adherence influenced the results. At present, additional research into the association between weight-bearing physical activity and DFU healing is needed.

\section{Offloading Adherence}

Consistent use of offloading footwear during weightbearing activity is a significant challenge [28, 31, 32]. Initial data concerning offloading adherence evolved out of studies regarding offloading devices used to treat active wounds. Historically, total contact casts had long been considered the gold standard amongst many options for offloading DFU [6]. However, in actual practice removable cast walkers have been used much more commonly [33]. While total contact casts and removable cast walkers have demonstrated similar capacities for offloading DFU, total contact casts have consistently demonstrated better healing outcomes in clinical trials [7]. Whereas removable cast walkers are readily able to be taken off and then reapplied at users' discretion, total contact casts must be cut in order to be removed. Thus, the difference in healing outcomes between the two options has historically been assumed to reflect insufficient wear-time of removable cast walkers.

The first study to objectively monitor offloading adherence used a dual accelerometer method to monitor the adherence of $20 \mathrm{DFU}$ patients with their removable cast walkers [31]. One accelerometer was worn on the participants' waists in order to quantify physical activity, while the second time-synchronized accelerometer was placed on the cast walker. Use of removable cast walkers was determined by assessing whether the cast walker accelerometers and waist-worn accelerometers simultaneously registered movement. On average, the study participants only wore their walkers for $28 \%$ of their daily steps. Following this initial adherence study, multiple studies evaluated whether rendering a removable cast walker irremovable (by wrapping cast tape or other material around it after it was applied) affected DFU healing [6]. These studies found that the healing efficacy of removable cast walkers was similar to that of total contact casts when the removable cast walkers were made irremovable. More recently, a previously referenced study was conducted to objectively monitor the association between varied adherence levels and DFU healing [28]. The study monitored offloading adherence and wound-healing progress for 79 individuals with DFU over a 6-week protocol. Multivariate analysis demonstrated that greater adherence significantly increased DFU healing rates.

In addition to studies investigating the importance of adherent use of offloading devices for healing DFU, a recent series of studies have objectively established deficiencies in adherent use of preventative offloading footwear. In 2012 Bus et al. [19] validated a temperaturebased technology for monitoring footwear usage. A thermal sensor located in the footwear used body heat to determine whether the footwear was being worn. A subsequent study that used the monitor with a sample of 107 
patients at risk of DFU found that only $29 \%$ of the patients adherently ( $>80 \%$ of daily steps) wore their prescribed offloading footwear, while $33 \%$ of the patients adhered for less than $60 \%$ of their daily steps [32]. Then, an additional study by Bus et al. [12] demonstrated the impact of low adherence. In that study patients with recently healed DFU were randomized to receive either standard custommade footwear or custom-made footwear that was optimized for offloading by modifying them based upon plantar pressure assessments. The initial intention-totreat analysis of 171 participants showed no difference in DFU recurrence between the two groups. However, when the analysis was limited to participants who wore their offloading device for $\geq 80 \%$ of their steps there was a significant reduction in DFU recurrence in the group that received the plantar pressure optimized footwear $(n=35)$ compared to standard custom-made footwear $(n=44)$. Thus, the authors concluded that the plantar pressure optimized footwear will not reduce DFU recurrence unless it is worn as recommended.

To date, objective monitoring of offloading adherence has been limited to research studies. Due to the growing body of literature substantiating the link between adherence and clinical outcomes, incorporation of offloading adherence monitoring has been recommended for DFU studies whenever possible [34]. However, benefits could likely be reaped from incorporating adherence monitoring into standard clinical care [35]. Both patients and care providers may modify their practices if provided objectively gathered data regarding patients' offloading practices. Aside from simply providing patients their adherence numbers, it may become practical to give alerts and other advice to patients in real time based upon their adherence/physical activity profiles. With the development of such capabilities will come debates as to whom should ultimately have access to the information collected [35]. There are numerous ethical and fiscal questions that will need to be considered. For example, should insurers be allowed to modify DFU care reimbursements based upon nonadherence? While caution is advised in assessing cost versus patient beneficence controversies, the provision of adherence data to care providers and patients would likely improve outcomes.

\section{Optimizing User Experience}

To date, much of the effort in evaluating and improving offloading devices has narrowly focused upon the devices' offloading profiles. Although adherent use of these devices is expected, the user experience with these devices is generally overlooked. In order to achieve better offloading outcomes, one needs to consider not only how design features impact foot loading but also what secondary effects the design features will impose upon the user. The best predictor of adherence identified in the previously referenced study [28], which demonstrated the positive association between offloading adherence and DFU healing, was patients' self-reported level of postural instability. Diabetes, aging, and falls are an interrelated troublesome trifecta [36]. Considering the ambulatory deficits associated with diabetic peripheral neuropathy (the primary risk factor for DFU) [37], it should not be surprising that DFU patients resist the use of cast walkers or other offloading modalities that may further alter their gait and balance. Although postural and/or gait instability increase the likelihood of losing balance, the ultimate cause of a fall is failure to initiate a proper recovery (compensatory) response to a loss of balance. This response can involve enacting a corrective ankle or hip torque, or if that proves insufficient, initiating a protective step. Peripheral sensation has been shown to alter compensatory stepping responses in healthy adults [38], and several studies on nondiabetic populations suggest that the offloading devices used to treat DFU could have detrimental consequences for compensatory responses. A study of healthy young adults found greater upper body motions during perturbed treadmill walking while wearing cast walkers compared to athletic shoes [39], thus implying worse stability. A common feature of offloading devices used to treat DFU, rocker bottom soles, have been found in nondiabetic patients to result in increased center of mass and center of pressure sway as well as delayed and longer response times following postural perturbations applied during quiet standing [39, 40]. A study that did not involve compensatory responses but did utilize individuals with active DFU, found that the use of a rubber heel with total contact casts resulted in greater postural sway during quiet standing [41]. Impaired postural control, as demonstrated by greater sway, may increase reliance on the stepping response, which may be compromised by neuropathy. Further research is needed regarding the potential influence of offloading devices upon compensatory responses of individuals with or at risk of DFU. Should offloading devices be shown to hinder the compensatory response in this patient population, secondary studies should evaluate whether physical training is capable of improving the compensatory stepping response and subsequently reducing fall risk in DFU patients using offloading devices. 
Such training has been shown to be beneficial in nondiabetic older adults [42].

Challenges to stability are not the only design issue that may impact user experience with offloading devices for active DFU. Despite a large number of available options to offload active DFU (in-shoe and ankle-foot orthoses, half-shoes, forefoot offloading shoes, ankle-high cast walkers, knee-high cast walkers, total contact casts, and others), the International Working Group on the Diabetic Foot recommends use of knee-high options [6]. While adherence is not considered to be an issue with the knee-high total contact casts, total contact casts are used minimally [33]. Knee-high removable cast walkers have rigid struts or circumferential encasements as well as padding material that run up the length of the shank. Thus, these are relatively heavy devices that the physically deconditioned [43] population of individuals with DFU are asked to wear at all weight-bearing times. A study comparing the offloading functionality of knee-, ankle-, and shoe-height cast walkers in individuals with diabetic peripheral neuropathy [44] found the shoe-height walker provided the least amount of offloading, while the ankleand knee-height walkers performed similarly. Considering the similar offloading profiles between knee- and ankle-height cast walkers, additional studies comparing the healing efficacy of the two devices are warranted. The smaller ankle-height devices may lead to greater adherence and ultimately better clinical outcomes. A recent, modestly sized randomized trial provides some credence to this theory [45]. The study randomized $60 \mathrm{DFU}$ patients to either a traditional knee-high total contact cast, an ankle-high cast walker rendered irremovable, or the same ankle-high cast walker left removable. Over a 90day protocol, there were no differences in DFU healing between the 3 groups. These findings differ from those of a similarly sized study in which a knee-high removable cast walker provided poorer healing than the same walker rendered irremovable [46]. The difference likely reflects greater use of ankle-high than knee-high removable walkers.

Regardless of total height, offloading devices used to treat DFU tend to induce artificial limb length discrepancies due to their thick soles that are often rocker bottoms. Limb length discrepancies have previously been associated with several musculoskeletal problems such as joint and back pain, declines in gait and balance, and increases in oxygen consumption and perceived exertion [47-49]. Two recent studies evaluated the use of a commercially available contralateral limb lift in tandem with a removable cast $[50,51]$. One found provision of the lift to the contralateral limb improved self-reported comfort of individuals at risk of DFU [50]. The other study found the lift counteracted changes in spinal alignment that were induced by the removable cast walker [51]. Taking into consideration not only what offloading options will work for DFU but also what options will work for patients may result in improved patient experiences and better wound healing.

Whereas offloading devices used to treat active DFU are expected to be worn temporarily, offloading devices for preventing DFU are intended to be worn as long as the user remains DFU free. Although they are generally less substantial in design and less disruptive to gait than offloading modalities used for active DFU, there is still substantial room for improvement in the eyes of users. Within their line of studies objectively monitoring adherence to preventative offloading devices, Waaijman et al. [32] also assessed predictors of adherence. In multivariate regression analysis they found perceived esthetics to be an important predictor of higher adherence. A study of 40 individuals with diabetes who had previously received a prescription for therapeutic footwear similarly identified style as the most common response to the question, "What are the most important things with regard to your footwear?" [52]. In a qualitative study regarding diabetic therapeutic footwear, Johnson et al. [53] noted a key finding was that patients and health professionals had differing perspectives in terms of expectations and reality of preventive behavior. Research has found an association between perceived benefit of diabetic shoes and adherent use of the shoes [54], suggesting improved education may have a role to play in improving adherence. Although a number of individual studies have reported style, perceived benefit, and other factors to be predictive of diabetic shoe adherence, a 2016 systematic review concluded that there are presently too few studies to draw any definitive conclusions [55]. Furthermore, the heavy reliance on self-reported measures of adherence in these studies underscores the need for additional research into predictors and modifiers of adherence.

\section{Conclusion}

DFU present a substantial burden to elderly individuals. Offloading is a key factor in healing active DFU and preventing new DFU from forming. Adherent use of offloading footwear remains a challenge, and improvements in the user experience with these devices may result in improved adherence. Other recent and forecasted fu- 
ture advances in offloading have relied heavily on technology to optimize devices' offloading capacity, provide objective feedback regarding users' physical activity profiles, and monitor tissue health via thermometry.

\section{Disclosure Statement}

The authors have no conflicts of interest to report in association with this study.

\section{References}

1 Zhang P, Lu J, Jing Y, Tang S, Zhu D, Bi Y: Global epidemiology of diabetic foot ulceration: a systematic review and meta-analysis dagger. Ann Med 2017;49:106-116.

-2 Armstrong DG, Boulton AJ, Bus SA: Diabetic foot ulcers and their recurrence. $\mathrm{N}$ Engl J Med 2017;376:2367-2375.

-3 Skrepnek GH, Mills JL Sr, Lavery LA, Armstrong DG: Health care service and outcomes among an estimated 6.7 million ambulatory care diabetic foot cases in the US. Diabetes Care 2017;40:936-942.

4 Margolis DJ, Malay DS, Hoffstad OJ, Leonard CE, MaCurdy T, Tan Y, Molina T, de Nava KL, Siegel KL: Economic Burden of Diabetic Foot Ulcers and Amputations: Data Points \#3. Rockville, Data Points Publication Series, 2011.

5 Wu SC, Crews RT, Armstrong DG: The pivotal role of offloading in the management of neuropathic foot ulceration. Curr Diab Rep 2005;5:423-429.

6 Bus SA, Armstrong DG, van Deursen RW, Lewis JE, Caravaggi CF, Cavanagh PR; International Working Group on the Diabetic Foot: IWGDF guidance on footwear and offloading interventions to prevent and heal foot ulcers in patients with diabetes. Diabetes Metab Res Rev 2016;32(suppl 1):25-36.

7 Bus SA, van Deursen RW, Armstrong DG, Lewis JE, Caravaggi CF, Cavanagh PR; International Working Group on the Diabetic Foot: Footwear and offloading interventions to prevent and heal foot ulcers and reduce plantar pressure in patients with diabetes: a systematic review. Diabetes Metab Res Rev 2016;32(suppl 1):99-118.

8 Wrobel JS, Najafi B: Diabetic foot biomechanics and gait dysfunction. J Diabetes Sci Technol 2010;4:833-845.

$\checkmark 9$ Bus SA: Foot structure and footwear prescription in diabetes mellitus. Diabetes Metab Res Rev 2008;24(suppl 1):S90-S95.

10 Gutekunst DJ, Hastings MK, Bohnert KL, Strube MJ, Sinacore DR: Removable cast walker boots yield greater forefoot off-loading than total contact casts. Clin Biomech (Bristol, Avon) 2011;26:649-654.

-11 Ulbrecht JS, Hurley T, Mauger DT, Cavanagh PR: Prevention of recurrent foot ulcers with plantar pressure-based in-shoe orthoses: the CareFUL prevention multicenter randomized controlled trial. Diabetes Care 2014;37: 1982-1989.
12 Bus SA, Waaijman R, Arts M, de Haart M, Busch-Westbroek T, van Baal J, Nollet F: Effect of custom-made footwear on foot ulcer recurrence in diabetes: a multicenter randomized controlled trial. Diabetes Care 2013;36: 4109-4116.

13 Lavery LA, LaFontaine J, Higgins KR, Lanctot DR, Constantinides G: Shear-reducing insoles to prevent foot ulceration in high-risk diabetic patients. Adv Skin Wound Care 2012;25:519-524; quiz 525-526.

14 Lavery LA, Higgins KR, La Fontaine J, Zamorano RG, Constantinides GP, Kim PJ: Randomised clinical trial to compare total contact casts, healing sandals and a shear-reducing removable boot to heal diabetic foot ulcers. Int Wound J 2015;12:710-715.

15 Frykberg RG, Gordon IL, Reyzelman AM, Cazzell SM, Fitzgerald RH, Rothenberg GM, Bloom JD, Petersen BJ, Linders DR, Nouvong A, Najafi B: Feasibility and efficacy of a smart mat technology to predict development of diabetic plantar ulcers. Diabetes Care 2017;40: 973-980.

16 Lavery LA, Higgins KR, Lanctot DR, Constantinides GP, Zamorano RG, Athanasiou KA, Armstrong DG, Agrawal CM: Preventing diabetic foot ulcer recurrence in high-risk patients: use of temperature monitoring as a self-assessment tool. Diabetes Care 2007;30: 14-20.

17 Skafjeld A, Iversen MM, Holme I, Ribu L, Hvaal K, Kilhovd BK: A pilot study testing the feasibility of skin temperature monitoring to reduce recurrent foot ulcers in patients with diabetes - a randomized controlled trial. BMC Endocr Disord 2015;15:55.

18 Najafi B, Mohseni H, Grewal GS, Talal TK, Menzies RA, Armstrong DG: An optical-fiber-based smart textile (smart socks) to manage biomechanical risk factors associated with diabetic foot amputation. J Diabetes Sci Technol 2017;11:668-677.

19 Bus SA, Waaijman R, Nollet F: New monitoring technology to objectively assess adherence to prescribed footwear and assistive devices during ambulatory activity. Arch Phys Med Rehabil 2012;93:2075-2079.

20 Maluf KS, Mueller MJ: Novel Award 2002. Comparison of physical activity and cumulative plantar tissue stress among subjects with and without diabetes mellitus and a history of recurrent plantar ulcers. Clin Biomech (Bristol, Avon) 2003;18:567-575.
21 Armstrong DG, Lavery LA, Holtz-Neiderer K, Mohler MJ, Wendel CS, Nixon BP, Boulton AJ: Variability in activity may precede diabetic foot ulceration. Diabetes Care 2004;27: 1980-1984.

22 Waaijman R, de Haart M, Arts ML, Wever D, Verlouw AJ, Nollet F, Bus SA: Risk factors for plantar foot ulcer recurrence in neuropathic diabetic patients. Diabetes Care 2014;37: 1697-1705.

23 Kluding PM, Bareiss SK, Hastings M, Marcus RL, Sinacore DR, Mueller MJ: Physical training and activity in people with diabetic peripheral neuropathy: paradigm shift. Phys Ther 2017;97:31-43.

24 Armstrong DG, Boulton AJ: Activity monitors: should we begin dosing activity as we dose a drug? J Am Podiatr Med Assoc 2001; 91:152-153.

25 Najafi B, Crews RT, Wrobel JS: Importance of time spent standing for those at risk of diabetic foot ulceration. Diabetes Care 2010;33. 2448-2450.

26 Crews RT, Yalla SV, Dhatt N, Burdi D, Hwang S: Monitoring location-specific physical activity via integration of accelerometry and geotechnology within patients with or at risk of diabetic foot ulcers: a technological report. J Diabetes Sci Technol 2017;11:899-903.

27 Vileikyte L, Shen BJ, Brown S, Boulton AJ, Kirsner R, Reeves ND, Crews RT: Depression, physical activity, and diabetic foot ulcer healing; American Diabetes Association 77th Scientific Sessions. Diabetes 2017;66(suppl 1): A168.

28 Crews RT, Shen BJ, Campbell L, Lamont PJ, Boulton AJ, Peyrot M, Kirsner RS, Vileikyte $\mathrm{L}$ : Role and determinants of adherence to offloading in diabetic foot ulcer healing: a prospective investigation. Diabetes Care 2016;39: 1371-1377.

29 Pence BD, Woods JA: Exercise, obesity, and cutaneous wound healing: evidence from rodent and human studies. Adv Wound Care (New Rochelle) 2014;3:71-79.

30 Najafi B, Grewal GS, Bharara M, Menzies R, Talal TK, Armstrong DG: Can't stand the pressure: the association between unprotected standing, walking, and wound healing in people with diabetes. J Diabetes Sci Technol 2017;11:657-667. 
- 31 Armstrong DG, Lavery LA, Kimbriel HR, Nixon BP, Boulton AJ: Activity patterns of patients with diabetic foot ulceration: patients with active ulceration may not adhere to a standard pressure off-loading regimen. Diabetes Care 2003;26:2595-2597.

- 32 Waaijman R, Keukenkamp R, de Haart M, Polomski WP, Nollet F, Bus SA: Adherence to wearing prescription custom-made footwear in patients with diabetes at high risk for plantar foot ulceration. Diabetes Care 2013;36: 1613-1618.

-33 Wu SC, Jensen JL, Weber AK, Robinson DE, Armstrong DG: Use of pressure offloading devices in diabetic foot ulcers: do we practice what we preach? Diabetes Care 2008;31: 2118-2119.

34 Crews RT, Shen BJ, Campbell L, Lamont PJ, Hardman M, Boulton AJ, Peyrot M, Kirsner RS, Vileikyte L: Response to Comment on Crews et al: Role and determinants of adherence to off-loading in diabetic foot ulcer healing: a prospective investigation. Diabetes Care 2016;39:1371-1377. Diabetes Care 2016;39: e222-e223.

35 Crews RT, Bowling FL, Boulton AJ: Controversies in off-loading: should big brother be watching? Curr Diab Rep 2009;9:417-419.

-36 Crews RT, Yalla SV, Fleischer AE, Wu SC: A growing troubling triad: diabetes, aging, and falls. J Aging Res 2013;342650.

-37 Brown SJ, Handsaker JC, Bowling FL, Boulton AJ, Reeves ND: Diabetic peripheral neuropathy compromises balance during daily activities. Diabetes Care 2015;38:1116-1122.

38 Mille ML, Rogers MW, Martinez K, Hedman LD, Johnson ME, Lord SR, Fitzpatrick RC: Thresholds for inducing protective stepping responses to external perturbations of human standing. J Neurophysiol 2003;90:666-674.
39 Goodworth AD, Kunsman M, DePietro V, LaPenta G, Miles K, Murphy J: Characterization of how a walking boot affects balance. J Prosthet Orthot 2014;26:6.

40 Kimel-Scott DR, Gulledge EN, Bolena RE, Albright BC: Kinematic analysis of postural reactions to a posterior translation in rocker bottom shoes in younger and older adults. Gait Posture 2014;39:86-90.

-41 Lavery LA, Fleishli JG, Laughlin TJ, Vela SA, Lavery DC, Armstrong DG: Is postural instability exacerbated by off-loading devices in high risk diabetics with foot ulcers? Ostomy Wound Manage 1998;44:26-32, 34.

42 Rosenblatt NJ, Marone J, Grabiner MD: Preventing trip-related falls by communitydwelling adults: a prospective study. J Am Geriatr Soc 2013;61:1629-1631.

43 Kanade RV, van Deursen RW, Harding K, Price P: Walking performance in people with diabetic neuropathy: benefits and threats. Diabetologia 2006;49:1747-1754.

44 Crews RT, Sayeed F, Najafi B: Impact of strut height on offloading capacity of removable cast walkers. Clin Biomech (Bristol, Avon) 2012;27:725-730.

45 Piaggesi A, Goretti C, Iacopi E, Clerici G, Romagnoli F, Toscanella F, Vermigli C: Comparison of removable and irremovable walking boot to total contact casting in offloading the neuropathic diabetic foot ulceration. Foot Ankle Int 2016;37:855-861.

46 Armstrong DG, Lavery LA, Wu S, Boulton AJ: Evaluation of removable and irremovable cast walkers in the healing of diabetic foot wounds: a randomized controlled trial. Diabetes Care 2005;28:551-554.

47 Gurney B, Mermier C, Robergs R, Gibson A, Rivero D: Effects of limb-length discrepancy on gait economy and lower-extremity muscle activity in older adults. J Bone Joint Surg Am 2001;83-A:907-915.
48 Defrin R, Benyamin SB, Aldubi RD, Pick CG: Conservative correction of leg-length discrepancies of $10 \mathrm{~mm}$ or less for the relief of chronic low back pain. Arch Phys Med Rehabil 2005;86:2075-2080.

49 Zhang S, Clowers KG, Powell D: Ground reaction force and 3D biomechanical characteristics of walking in short-leg walkers. Gait Posture 2006;24:487-492.

50 R.T. C., Candela J, Ghazizadeh R: Offloading and comfort responses to walking in different heighted removable cast walkers with and without a contralateral limb length equalizer; American Diabetes Association 73rd Scientific Sessions. Diabetes 2013;62(suppl 1):A31.

51 Crews RT, Girgis C, Domijancic R, Mosher E, Yalla SV: Influence of offloading induced limb length discrepancies upon spinal alignment; American Diabetes Association 77th Scientific Sessions. Diabetes 2017;66(suppl 1): A27.

52 Williams AE, Nester CJ: Patient perceptions of stock footwear design features. Prosthet Orthot Int 2006;30:61-71.

53 Johnson M, Newton P, Goyder E: Patient and professional perspectives on prescribed therapeutic footwear for people with diabetes: a vignette study. Patient Educ Couns 2006;64: 167-172.

54 Arts ML, de Haart M, Bus SA, Bakker JP, Hacking HG, Nollet F: Perceived usability and use of custom-made footwear in diabetic patients at high risk for foot ulceration. J Rehabil Med 2014;46:357-362.

55 Jarl G, Lundqvist LO: Adherence to wearing therapeutic shoes among people with diabetes: a systematic review and reflections. $\mathrm{Pa}-$ tient Prefer Adherence 2016;10:1521-1528. 